The annual incidence of leukaemias in children is 4.5 cases per 100,000 children. Acute lymphoblastic leukaemia accounts for approximately $77 \%$ of cases. The common presentations in this age group are pallor, irritability and bone pain. Leukaemia cutis can be a rare presenting sign of ALL (3\% of cases).

\section{P568 GITELMAN SYNDROME: A RARE CAUSE OF HYPOKALEMIA}

Jan Roux, Amani Abdalla*, Muhammad Pervaiz. University Hospital Kerry, Tralee, Ireland

10.1136/archdischild-2019-epa.902

Gitelman syndrome is a rare autosomal recessive renal tubular disorder characterized by hypoglycemia, metabolic alkalosis, hypomagnesemia and hypocalciurea, most often it is caused by a mutation in the solute carrier family 21 member 3 (SLC12A3) gene. Prevalence is approximately 1:40000 and the prevalence of hetero zygotes is approximately $1 \%$ in Caucasian populations, making it one of the most inherited renal tubular disorders.

We report a case of 7 year old boy who presented to emergency department with 4 days history of abdominal pain.

Past history revealed that he always likes salty and pickled food, having intermittent weakness and low energy but no paraesthesia or muscle cramps.

He was born at term with uneventful neonatal course. His development is normal and is fully vaccinated.

There is no family history of renal disease, Dad has IDDM, and he has one healthy brother.

On examination he was a slim boy, weight was $21.2 \mathrm{~kg}$, height $119 \mathrm{~cm}$ (both between 9-25th centile) his vitals including blood pressure were normal. Systemic examination was normal apart from mild peri-umbilical tenderness. Abdominal X-ray showed faecal loading of colon and us abdomen was normal. He was referred for paediatric assessment by surgical team because of incidental finding of low serum potassium. His venous blood gas showed metabolic alkalosis and serum magnesium was low normal. ECG showed sinus rhythm.

He was admitted and started on intravenous fluids with added potassium chloride as well as oral $\mathrm{K}$ supplements.

Other investigations including early morning urine, albumin lcreatinine ratio, urine for electrolytes, urine amino acids and urine for retinol binding proteins (RBP) were all normal. Blood sample for genetics was sent for Gitelman syndrome.

His oral potassium dose was increased gradually while weaning on potassium supplement in intravenous fluids. $\mathrm{He}$ was discharged home on oral potassium after his serum potassium level improved and booked for repeat bloods in day ward. His repeat blood showed low serum magnesium, so he started on oral magnesium supplement. He also needed further increase in his potassium dose to maintain his serum level $\geq 2.8 \mathrm{mmol} / \mathrm{L}$. His genetics tests confirmed two mutations in the SLC12A3 gene consistent with autosomal recessive Gitelman Syndrome.

Currently he is doing well and under regular follow up with general paediatric and paediatric nephrology.

Although it is a rare disorder, Gitelman Syndrome should be considered in children with unexplained hypokalaemia, with adequate treatment patients usually have excellent prognosis.

\section{P569} DEJERINE-SOTTAS SYNDROME AND CRANIO-FACIAL DYSMORPHISMS: A CASE REPORT

${ }^{1}$ Alessandra Biagini*, 'Elena Nechita, ${ }^{2}$ Willie Reardon, 'Hilary Greaney, 'Ghia Harrison.

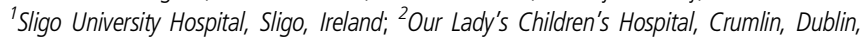
Ireland

10.1136/archdischild-2019-epa.903

Introduction Dejerine-Sottas syndrome (DSS) is a rare hereditary motor-sensor neuropathy transmitted as either autosomal dominant or recessive and classified as a severe degenerative neuropathy of the Charcot-Marie-Tooth type.

DSS is characterized by demyelination and remyelination features with an extensive nerve and root hypertrophy that results in a decreased nerve conduction velocity $(<10-12 \mathrm{~m} /$ s).

The hallmark clinical manifestations develop in early infancy with hypotonia, developmental motor delay and areflexia. Although arthrogryposis and spine deformities are frequent features, there are no direct associations with other dysmorphic features.

Case This report describes a rare association between DSS and cranio facial syndrome.

A 5 months old boy first presented in our Clinic with an early onset of motor symptoms manifested by congenital hypotonia, joint laxity particularly involving his lower limbs, failure to thrive, short stature and a significant psychomotor developmental delay.

On clinical examination he showed clear dysmorphic features with epicantic folds, hypertelorism, long philtrum, low set ears, downstanding of the eyes particularly the left eye and a convergent left eye squint.

Comment The presence of 'soft' clinical signs can distract from typical features of an underlying neurological syndrome leading to subsequent delayed or misdiagnosis in children with DSS.

In our experience, it is therefore important that Paediatricians can be aware of this possible association with this diagnosis and seek expert specialist geneticist advice if suspicious while simultaneously developing a management plan that supports and encourages attainment of maximal developmental process for the child.

\section{P570 AN UNUSUAL CAUSE OF RECURRENT VOMITING IN A SCHOOLBOY: DIETL'S CRISIS}

Ronan Callanan*, Alwyn Charles, Shozab Taj, Eoin Fitzgerald, Anne-Marie Murphy. UHL, Limerick, Ireland

\subsection{6/archdischild-2019-epa.904}

Backdround Congenital Pelvic Ureteric Junction Obstruction (PUJO) is caused by an intrinsic stenosis of the PUJ or external compression by an accessory renal artery.

Dietl's crisis is defined as episodic crampy upper abdominal pain, nausea and vomiting secondary to PUJO. Symptoms can be severe, increasing in nature with diuresis. The patient may also be asymptomatic between events.

Childhood vomiting is a regular presentation to Paediatric Emergency Departments (PED) worldwide

Aims Our aim is to report a case of an eight year old boy who presented to our PED with recurrent episodes of vomiting and abdominal pain, the ultimate diagnosis for which was Dietl's crisis. 